\title{
Roadmap Towards a Bioenergy Model of Life Cycle Sustainability Assessment
}

\author{
Eniko KOVACS ${ }^{1,2}$, Lacrimioara SENILA², Maria-Alexandra HOAGHIA ${ }^{2}$, Cecilia ROMAN², \\ Diana E. DUMITRAS 1
}

\author{
${ }^{1}$ Department of Economic Sciences, University of Agricultural Sciences and Veterinary Medicine, Cluj-Napoca, \\ Romania \\ 2 INCDO-INOE 2000, Research Institute for Analytical Instrumentation, Cluj-Napoca, Romania \\ *Corresponding author: D.E. Dumitrase-mail: ddumitras@usamvcluj.ro
}

\section{REVIEW}

\begin{abstract}
The emergent life cycle assessment (LCA) methodology, techniques and models were punctuated by divergences concerning the system expansion, allocation rules and causation modeling. Moreover, the unification of the economic, social and ecological perspectives in the life cycle sustainability assessment (LCSA) should be addressed by abstract models providing coherence and normalization. The purpose of this study was to identify, in the context of biomass waste processing into biofuels, some of the most representative generic and specific issues and theoretical gaps encountered in LCA and LCSA modeling, and to synthesize a list of requirements by analyzing some of the most consistent state of the art solutions, in order to develop an abstract LCSA model. The literature review covered selected studies on LCSA and biomass to biofuels and lignocellulosic agricultural waste valorization LCA techniques. A list of requirements resulted from the significant approaches, in support of a formal model to be developed.
\end{abstract}

Keywords: agricultural biomass waste; biofuels; ecology; modeling.

Received: 01 September 2020 Accepted: 13 December 2020 Published: 14 May 2021

DOI:

15835/buasvmen-hort:2020.0029

\footnotetext{
(ब) $\Theta \Theta \Theta(\odot) 2021$ Authors. The papers published in this journal are licensed under the Creative Commons Attribution-NonCommercialNoDerivatives 4.0 International License
}

\section{INTRODUCTION}

Industrial ecology (IE) transposes concepts from ecological systems towards industrial and urban systems, attempting to operationalize sustainability concepts through ecological-social-economic (the "triple bottom line" perspective) impact assessment methods, models, tools and frameworks. The IE systems approach is commonly applied to the environmental analysis, management and policy via the lifecycle assessment (LCA) framework, which combines inventory analysis (input-output material-energy flows, such as resources, products or by-products assigned to processes) and impacts analysis (potential or concrete effects on ecological, social or economic dimensions, as impact categories). LCA characterizes a production and consumption system from its environmental interactions perspective, along the value chain. The development of LCA methodology, techniques and models met conceptual hesitations starting with the foundations: system boundary, impact potentials and characterization factors, midpoint and endpoint impact categories The allocation rules controversies in the attributional LCA, the complexity of the equilibrium and optimization models in the consequential LCA and the issues related to the unification of the economic, social and ecological perspectives in the life cycle sustainability assessment (LCSA) call for a generic methodology based on 
abstract, coherentand normalized models. The LCA methodology comprises: (1) a goal and scoping phase, with the objective setting and the analysis concern, the functional unit and system limits definition; (2) the life cycle inventory phase (LCI), with a system partitioning in unit processes and associated material and energy flows; (3) the life cycle impact analysis (LCIA), mapping the unit flows' impact potentials to the midpoint (intermediate, with direct causation) and endpoint (final, with implicit causation) impact categories by assigning impact coefficients (characterization factors), then weighing and normalizing for the total impact calculation; (4) finally, the results interpretation phase (Figure 1). Defined as a framework, the LCA can integrate multiple modeling choices and data sources, but it necessitates a thorough evaluation of the scenarios consistency.

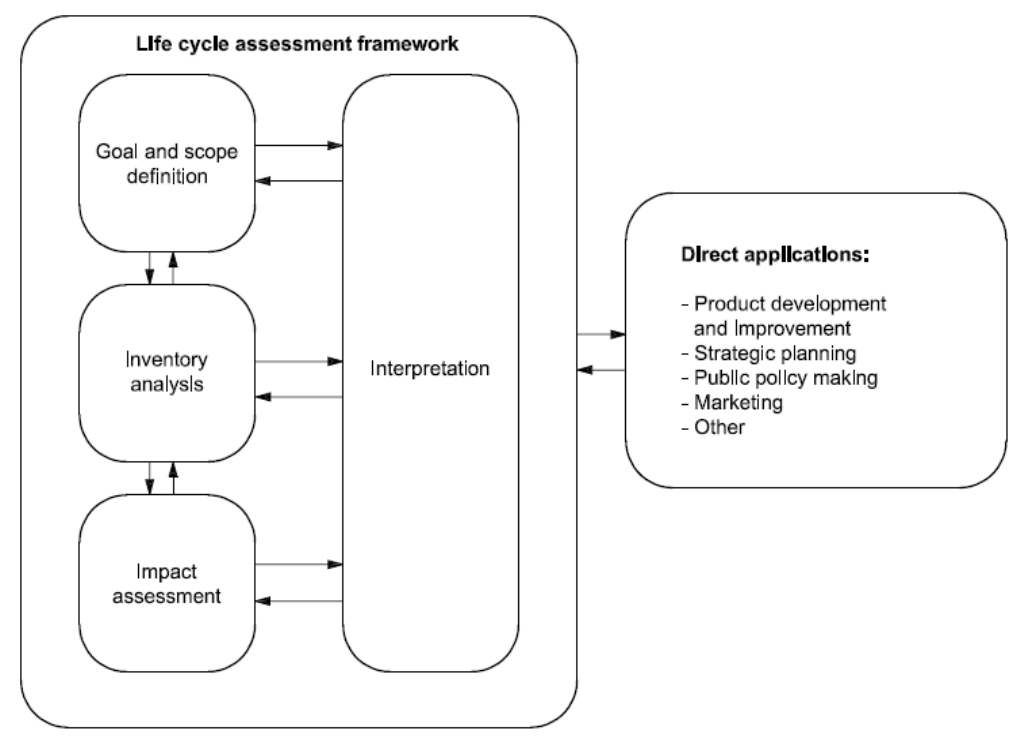

Figure 1. LCA phases (Source: ISO 14040:2006)

A conventional LCSA typically includes environmental life cycle assessment (ELCA), social LCA (SLCA), life cycle costing (LCC) and, according to the circumstances, other LCA variants, such as consequential, dynamic and hybrid approaches, and delivers an index composed of outputs from the environmental, social and economic analysis, but lacking of real integration, as shown by Ekener et al. 2018. Economic assessments in LCSA are justified only when validating technology effectiveness (capacity to deliver and resource efficiency) and for accounting on current and future societal costs. The prioritization of sustainability indicators uses the multi-criteria decision analysis (MCDA) methodology, mostly based on performance and preference, which has many variants, among which: Analytical Hierarchy Process (AHP), Dominance based Rough Set Approach (DRSA), Multi-Attribute Value Theory (MAVT), Multi Attribute Utility Theory (MAUT), Elimination and Choice Expressing the Reality (ELECTRE), Preference ranking Organization method for Enrichment of Evaluations (PROMETHEE).

The purpose of this study was to identify and discuss, in the context of lignocellulosic agricultural biomass waste processing into biofuels, the issues and theoretical gaps encountered in LCSA modeling, and to synthesize a list of requirements by analysing some of the most consistent current solutions (state of the art), in order to develop a new model of LCSA, with a higher abstraction level. A literature review supported the solutions and gaps analysis and the requirements collection for the LCSA model development, focusing on the lignocellulosic agricultural waste biomass processing. The LCSA model requirements were synthesized from selected studies on attributional and consequential LCA, theoretical developments on LCSA, residual lignocellulosic biomass processing, BtB LCA methodology and techniques. The analysis integrated complex modeling solutions to be considered in order to develop an LCSA model and to test it against the residual biomass processing context.

\section{MATERIALS AND METHODS}

\section{A review of conceptual advances in LCSA}

Central to the sustainability models operationalization (through LCSA) are: 1) the intrinsic valuation versus anthropic cultural perspectives (monetization) related to the natural resources and processes evaluation, and to the quantification of their interactions with the anthropic cultural system, which represents a component (competing for resources) of the "users system" in a socio-ecological system (SES); 2) the causalities (thermodynamic, chemical, biological and cultural) integration in ecological and social-economic networks (an 
agency definition problem) and the unified representation of the conventionally separated ecological, social and economic systems, and 3) the identification of goal functions describing an assemblage-level fitness concept and a system-level sustainability concept (avoiding the burden of a classical multi-agents optimization problem), and determining inter-components tradeoff algorithms shaping the network.

In their study, Millward-Hopkins et al. (2018) elaborate a model focused on substituting the monetary value with a generic currency, more appropriate for refining the materials valuation and improving waste resources value retention. Their model for renewable resources from waste (RRfW) value retention promotes the resource's technical value, based on embodied concentration values, as a causal driver (chemical determinism) of the mutual cumulative impacts resulting from transactions and relations between foreground and background systems. Along the material flows, the value's life cycle disaggregates following a hierarchical causal path, deciding between the end-of-life strategies (recovery or disposal). The "Complex-Value Optimization for Resource Recovery" (C-VORR) model gains in consistency by multiplexing the ecological, social and economic flows in a unique conceptual representation, instead of treating them separately and only weighting their indicators' results in a unique index, by converting the domain-specific metrics to metadata, at a higher abstraction level. Since the conventional direct allocation is not relevant, they use a consequential LCA approach to conceptualize the complex dynamics, with consistent system boundaries and using a whole spectrum value chain (with possible synergies). The model is grounded on their evaluation of the state-of-the-art, concluding that the LCSA should combine consistent and computable models with qualitative formalisms, being more analytical than extensive and using a holistic (aggregation-based), dynamic and anticipative (forecasts-based) approach, accounting for systemic reconfigurations' causal dependencies.

A social-material network analyses framework is proposed by Schiller et al. (2014) in order to integrate the cultural determinism with the economic behavior of the entities relaying and amplifying the network flows (e.g. financial transactions, energy and information exchange, social interactions). They elaborate on a conceptual representation of the anthropic cultural network, based on mapping physical connections (functional metabolism) to multi-layered social connections (e.g. contracts, informal agreements) between the social-economic entities. Social relationships mediate in large proportions the determinism of cultural and biophysical flows (e.g. knowledge, payments, materials, technology). The economic entity's sub-net, propagating social-cultural types of relations (e.g. industrial symbiosis, competition, corporate responsibility, technological and organizational alliances), together with the market constraints, determine not only the entity's transactions (material flows), but also its evolutionary processes (e.g. its cultural-organizational selection and diversity, its adaptation and speciation). This approach exploits the LCA potential for evaluating the social-economic networks' resilience and the stability of their ecoindustrial paths (e.g. industrial symbiosis), based on a further ecological conceptualization of their bio-physical resources base, in the context of the underlying social network and market structures. Authors suggest a combination of models of social-economic reciprocation, like the "symbiotic reverse flows" (local feedback loops) and the "socially nested production structures" (inter-scales feedback loops), as more relevant than a model of profit-based preferential attachment (in self-organizing and scale-free networks) for explaining the socialenvironmental metabolism's cumulative upward causation in SESs networks.

The thermodynamic determinism modeling proposed by the theoretical ecology was adopted by the industrial ecology through various models and indicators based on energy quality orientors (extremal principles, goal functions). Emergy and exergy can be mentioned among the orientors which were effectively integrated in both conventional and consequential LCA approaches, as indicators for sustainability or eco-efficiency evaluation and resources valuation, due to their general applicability and to their aggregation or scaling simplicity, facilitating both the gradients construction and systems comparison.

Based on the emergy calculation, socio-ecological systems (SES) can be compared, at different spatial-temporal scales and organizational levels, by capturing the value of the energy and material flows across the biosphere and technosphere, as explained by Brown and Ulgiati (1999). Ecosystems persistence and adaptive capacity are determined by the coherence of the cyclical processes alternating the energy concentration and dispersal phases via material flows, including biotic dissipative structures. SESs economic processes transfer energy from the natural storages, generating positive feedbacks in the technosphere, which are in essence increasingly decoupled from the ecosystems adaptive cycles and biogeochemical cycles, and thus from the natural processes coherence. Technosphere feedbacks are essentially regulated by financial objectives maximizing via market-related currencies, often detrimental to the self-organizing capabilities of the ecosystems. Beyond their speculative or arbitrary value (depending on an anthropocentric cultural determinism), market currencies might be used for measuring the value of the energy produced or exchanged within the economic domain. However, except the human economic behavior, they are not an appropriate instrument for quantifying the value of the flows occurring in the social and ecological domains. Energy (as quantitative thermodynamic variable) and its market price are either not relevant or not sufficient to characterize the biosphere's value chains: in natural complex adaptive systems, the biophysical structures conveying the flows reach higher technical levels and potentials than those created or replicated in the techno-sphere. Emergy adopts a qualitative vision on the energy, from the donor perspective, accounting for the 
total solar energy extracted over time from Nature, through all channels, and concentrated (embodied) into the considered resource. A candidate emergy indicator for integration with the LCSA framework would be the Emergy Sustainability Index, synthesizing environmental load, renewability and efficiency, based on the comparison of the emergy yield ratio (EYR, the total emergy loss per unit of emergy input) with the environmental loading ratio (ELR, the total nonrenewable and imported emergy inputs per unit of locally produced renewable resource).

Exergy, another indicator used by the LCA studies and derived from the $2^{\text {nd }}$ law of thermodynamics, is a measure of the materials' energetic quality degradation during the dissipative conversion processes, as stated by Zvlolinschi et al. (2007). The authors suggest the following exergy indicators for evaluating and comparing the sustainability of the energy conversion systems' metabolisms: the exergy renewability ratio, the exergy efficiency and the exergy used for emissions abatement and materials recycling. They conclude that exergy loss (entropy production) can be minimized, although exergy is not completely renewable via multilevel material cycles. Gulotta et al. (2018) proposed a new series of indicators, derived from the Cumulative Exergy Demand (CExD), which measures the destroyed exergy, being used in LCA comparisons of technology systems. CExD denotes the sum of the exergies of all resources (or inputs, including fuels and chemical potential) required to provide a process or product. The new indicators are the Useful Cumulative Exergy (UCEx) and the Life Cycle Irreversibility Index (LCII). UCEx, the exergy of the system outputs, is used to define the system efficiency, as the ratio of UCEx and CExD. The LCII measures the technology obsolescence, as a ratio between the UCEx of a real system and the UCEx of the best available technologies (BAT) or of functionally equivalent theoretical systems, revealing the inefficiency of technical processes.

Exergy-emergy ratio was suggested by Bastianoni (2002) to measure the ecosystem efficiency in self-organizing and in generating complex functional structures. Adapted to the context, the exergy measures the energy of the selforganizing natural recycling processes (biogeochemical cycles), while the emergy measures the energy complexity of natural components, as they recursively embody other energy carriers (natural components themselves) along their life cycle. Recursivity leads back to the primary biomass producers in the trophic chain, therefore total emergy is expressed in solar energy, ideal for weighting and normalization.

\section{A review of LCA operational techniques and issues}

A series of inconsistencies were observed in LCSA modeling, regarding the impacts quantification, weighting and normalisation (monetary versus intrinsic ecological and social valuation), the indirect effects, the co-evolutionary aspects of a system, the lack of social and bio-ecological causations. Besides, determining homogenous methods for harming potential and realized damage evaluation, and their integration in a predictive coherent behavioral model remain major challenges.

Benetto et al. (2015) observed that LCA methodology supports decision making strategies, but the unavailability of the environmental profile for certain impact categories, the choice of different system boundaries and the consideration of system expansion may limit LCAs comparison. Data can be collected either from empirical studies or from the technical infrastructure specifications or extracted from the LCA software/methodology database (for instance: GaBi6, SimaPro-Ecoinvent, Greet). For the overall impact assessment calculation, endpoint results' normalization factors (ReCiPe method) are expressed in "points" (equivalent to [persons-year] ${ }^{-1}$ ). Impacts are aggregated in three categories (human health, ecosystem quality and resources) and weighted according to the hierarchist approach (average scheme: $40 \%$ human health, 40\% ecosystem quality and 20\% resources, according to $\mathrm{ReCiPe}$ ) or to the mixing triangle approach (an alternative is the use of multi-criteria decision aiding methods). Marvuglia et al., 2013 defined the scope of the two fundamental LCA methodologies, as follows: the attributional life cycle assessment (ALCA) estimates the average environmental impact per functional unit produced using a given technology, by calculating the mass and energy flows' average inventories, in a steady state market (context independence), while the consequential life cycle assessment (CLCA) estimates the scale-dependent and dynamic environmental impacts of variable technological implementations and market contexts, under regulating constraints, by capturing marginal changes' direct and indirect causations (context dependence, i.e. social-economic influence) applied to the equilibrium of the technical system defined by the ALCA. As stated by Roos et al. (2018), the CLCA approach considers the external consequences of changes and decisions, in particular their environmental effects, by exploring alternatives for system boundaries and by combining different aggregation and complexity levels. It integrates economic equilibrium or optimization and causal modeling, seeking for methodological diversity; it uses marginal instead of average figures and it benefits from abstract methods based on decision-trees (algorithms) for the stepwise identification of the affected processes. CLCA scenario modeling calls for a multi-model approach, where all constraints have to be modeled in CLCI. A sound solution is adopting basic system boundaries by using ALCA as first approach, before investigating causalities, including scale effects and time horizons, with CLCA (using alternative definitions of system boundaries and modeling). An ALCA characterizes the potential environmental impacts attributed to a product system over its life cycle (upstream, along the supply-chain, and downstream, following the end-of-life value chain), as McManus and Taylor (2015) observed.

In biomass to biofuels (BtB) LCAs, distinct scenarios correspond to combinations between processes site locations (centralized or decentralized) and energy source types (renewable or non-renewable). A decentralized architecture 
leads to reduced impacts, depending on the transport distances, and on the equipment downscaling (Kylili et al., 2016). Biofuels and conventional fuels technological systems can also be compared. LCIA consistency checks can be performed with the following parameters: drying fuel type, multi-functionality approach and water content variability. Drying scenarios could distinguish the energy carriers (natural gas, electricity and wood chips). Multi-functionality can be handled in several scenarios: Cut-Off, Mass Allocation, Economic Allocation and System Expansion. In the CutOff approach, no environmental burden or credit is allocated to neutral products. In the Mass Allocation case, a fraction of the environmental impacts (proportional to the output-input mass ratio) of the process is allocated to the output. In the case of Economic Allocation, the fraction of the environmental impacts attributed to the output is proportional to the forecasted revenue generated. The expanded system has high environmental impacts variability: significant reduction of impact is due to the credits allowed (Benetto et al., 2015). For waste valorization, a good choice is a functional unit based on the amount of waste biomass valorized per batch and considering multi-output system scenarios for comparison. Functional unit derived from the production amount and potential revenues compensate the worse environmental effects of the intensive stages by the value added (Gullon et al., 2018). Common system boundary setting methods are: cut-off, allocation and system expansion. The co-products are managed by cut-off or system expansion (Benetto et al., 2015).

Bio-refinery strategies for closing the loop (recycling and re-using waste streams) worsen the production systems' environmental profiles related to many impact categories, due to the increased chemicals and energy requirements (Gullon et al., 2018). The purity of the final product has an influence on the waste valorization (Benetto et al., 2015). The exploitation of renewable energy sources for energy generation and consumption contribute to the impact reduction (Kylili et al., 2016). Process infrastructures are out of the scope. In agricultural waste valorization, carbon imbalances are cut-off (Benetto et al., 2015). Pellets from agricultural waste avoid direct pollutant emissions from UoL, but it also reduces the benefit of maintaining organic matter and nutrients cycling in soil, necessary for the culture (Gullon et al., 2018). Ashes produced during pellets combustion may have either the status of waste or valuable products, depending on their market price, which characterizes the ability of the system to sustain their production (Benetto et al., 2015).

The bioenergy global environmental impact is mainly due to: biomass cultivation (land use has a large contribution, together with surface used in energy crops production, fossil fuels consumption for fertilizer production and nitrogen oxides emissions due to the urea application) and wood pelleting (mainly due to diesel and corn starch consumption). Among the macro-processes, contributions to the single score impact are derived from pellet combustion and from cultivation-related fertilizers production (Fantozzi, and Buratti, 2010). The impact categories having the highest contribution to the single score impact, after normalization and weighting, are human toxicity, marine eco-toxicity, freshwater eutrophication and natural land transformation. At the endpoint level, heat production from biomass waste is preferable to alternative fuels, the main contributors to the environmental impacts being the combustion of pellets and the drying phase (Benetto et al., 2015). Agricultural operations have a large contribution to the environmental impact, when evaluated with both EcoIndicator 99 and EPS 2000, while EDIP results are flawed due to the weight of the infrastructure and machinery construction (Fantozzi, and Buratti, 2010).

\section{Collected requirements for an integrated LCSA modeling}

Four categories of generic modeling requirements were identified, corresponding to the: LCA theoretical gaps and state of the art, and to LCSA theoretical gaps and state of the art, and are listed below. The reviewed studies identified some of the LCA current theoretical gaps to be covered by a LCSA generic model (Table 1).

Table 1. Generic modeling requirements from LCA theoretical gaps Generic modeling
requirements from LCA
theoretical gaps

\begin{tabular}{l}
$\begin{array}{l}\text { Abstract methods based on decision-trees (algorithms) for the stepwise identification } \\
\text { of the affected processes }\end{array}$ \\
\hline Multi-model approaches integrating all the constraints \\
\hline Environmental profiles for certain impact categories \\
\hline Rules for system expansion, facilitating LCAs comparison
\end{tabular}

LCA modeling state of the art comprises the following points to be considered by further modeling initiatives and possibly enhanced (Table 2).

Table 2. Generic modeling requirements from LCA state of the art

Generic modeling requirements from LCA state of the art
ALCA estimates the average environmental impact per functional unit produced using a given technology, by calculating the mass and energy flows' average inventories, in a steady state of the system 
CLCA estimates the scale-dependent and dynamic environmental impacts of variable technological implementations and market contexts, under regulating constraints, by capturing marginal changes' direct and indirect causations (context dependence, i.e. socialeconomic influence) applied to the technical system defined by the ALCA

CLCA approach considers: external consequences of changes; alternatives for system boundaries; different aggregation and complexity levels; economic equilibrium, optimization and causal modeling; marginal instead of average figures; causalities investigation, including scale effects and time horizons

In the ReCiPe method, the overall impact is calculated through midpoint values normalization (equivalent to [persons-year] ${ }^{-1}$, expressed in points), weighting (according to the cultural theory of risk, e.g. egalitarian, hierarchist, individualist, fatalist) and aggregated into endpoint categories (human health, ecosystem quality and resources)

Data can be collected from empirical studies, technical infrastructure specifications or extracted from databases

The reviewed studies also identified some of the LCSA current theoretical gaps, which are requirements of the highest priority for an enhanced LCSA model (Table 3).

Table 3. Generic modeling requirements from LCSA theoretical gaps

\begin{tabular}{|c|c|}
\hline \multirow{9}{*}{$\begin{array}{l}\text { Generic modeling } \\
\text { requirements from } \\
\text { LCSA theoretical gaps }\end{array}$} & $\begin{array}{l}\text { Outputs are composite indicators resulting from inconsistent combinations of category } \\
\text { indicators of parallel models, leaving sustainability perspectives disparate and lacking of } \\
\text { final integration }\end{array}$ \\
\hline & $\begin{array}{l}\text { Integrated environmental/economic indicators are missing, therefore aggregation is } \\
\text { problematic }\end{array}$ \\
\hline & $\begin{array}{l}\text { Dynamic approaches (temporal projections) are missing, the focus being on the process } \\
\text { downstream, eluding entropy prevention strategies }\end{array}$ \\
\hline & System boundaries are arbitrarily established, without causal dependencies analysis \\
\hline & $\begin{array}{l}\text { The organizations' social interactions role, as determinant of the functional metabolism, is } \\
\text { not considered }\end{array}$ \\
\hline & $\begin{array}{l}\text { The organizations' social interactions role, as determinant of the functional metabolism, is } \\
\text { not considered }\end{array}$ \\
\hline & $\begin{array}{l}\text { Ecology concepts were transposed to the market models, but not extended further to the } \\
\text { non-market social structures of the system's network }\end{array}$ \\
\hline & $\begin{array}{l}\text { The socio-environmental metabolism's cumulative upward causations' propagation are } \\
\text { not formalized }\end{array}$ \\
\hline & $\begin{array}{l}\text { Self-organizing and scale-free networks based on preferential attachment do not explain } \\
\text { the social evolutionary mechanisms }\end{array}$ \\
\hline
\end{tabular}

LCSA modeling state of the art (according to the literature review) suggests the following features to build on when developing a generic model applicable to SESs (Table 4).

A generic model can be adjusted to fit any specific scenario, by considering specific (local) variables. In biomass to biofuels (BtB) LCAs, the most influential factors, at the scenario level (lignocellulosic waste valorization), were also extracted from the state of the art (Table 5).

A list of requirements can be realized in order to further specify an extension of the generic model, not only adjusted to the BtB scenarios, but also to specific agricultural waste valorization, such as the vine shoot waste resulted from vineyard pruning operations.

Based on the state of the art solutions and identified gaps, a "total ecosystem model" (TEM) for LCSA will be specified in a future work, including a conceptual model for an extended ecosystem (a homogenous representation of the social-ecological systems' components and interactions) and two scorecards (containing metrics for sustainability and fitness) measuring the extended ecosystem's performance in terms of "naturalness".

A process-oriented model, seeking for consistency between the goal-functions associated to the system's components (individual fitness) and the system's sustainability constraints (expressed as orientors), can be integrated with the LCA framework. Phenotypes or entities of cultural or biological species, as collections of processes, are receiving, buffering and flowing energy-matter-information throughout the network, being the main causal relay in a LCA system modeled as an ecosystem. 
Table 4. Generic modeling requirements from LCSA state of the art

Integrating the environmental, social, economic and technical perspectives with MFA and LCA methodologies

Converging concepts into a unique construct and delivering a one-dimensional, consistent and computable multiplex model.

Representing systems via multiplexing physical and social networks capturing the multilayered connections between the social-economic entities: energy flows, financial transactions, information and social interactions

Forecasting the causal projections (in time and space) of the anthropogenic dissipative physical flows mediated by social relationships

Considering the economic entities' social connectivity (networks) and the market constraints among the SES's evolutionary states selectors, including symbiotic flows (proximity feedback loops) and nested production structures (inter-scales feedback loops)

Converting domain specific metrics to an abstract and consistent multi-domain analysis typology.

Generic modeling requirements from LCSA state of the art

Demonstrating direct and indirect social, environmental and economic interactions mediated by a resource's technical value fluctuations

Considering resource's value retention and optimization; computing a hierarchical path for the material flows' technical value, where transfer coefficients over the chemicals' life cycle stages are based on concentrations

Considering embodied values describing mutual cumulative impacts between the system and its environment.

Embracing a consistent approach with respect to the system boundaries; capturing the full values spectrum including qualitative metrics

Capturing the intrinsic technical value of energy/material flows and natural biophysical structures across the biosphere, from the resource donor perspective, via the SESs emergy evaluation at different organizational levels and spatial-temporal scales

Calculating exergy-emergy efficiency ratio reflecting the ecosystem's self-organization (exergy) and complexity (emergy) levels along its life cycle

Evaluating materials' energetic quality degradation, during the dissipative conversion processes, reflected by the exergy loss

Comparing the conversion systems' metabolism, in particular real technical systems against best available technologies (BAT), for similar function and scale, in order to limit the exergy loss and the entropy production

Table 5. Scenario specific modeling requirements from BtB case studies

\begin{tabular}{ll}
\hline & $\begin{array}{l}\text { Centralized or decentralized sites, determining the required equipment and transport } \\
\text { distances }\end{array}$ \\
\cline { 2 - 2 } & $\begin{array}{l}\text { Energy source type (renewable or non-renewable) fuelling the technology systems (e.g. } \\
\text { drying fuel type: natural gas, electricity, wood chips) }\end{array}$ \\
\hline $\begin{array}{l}\text { Multi-functionality handling rules: cut-off (co-products are neutral), mass allocation } \\
\text { (fractional attribution based on input-output mass ratio), economic allocation (fractional } \\
\text { attribution based on revenues forecast), and system expansion (avoidance credits allowed) }\end{array}$ & $\begin{array}{l}\text { Waste valorization functional unit choice (amount of biomass waste valorized in multi- } \\
\text { output system scenarios versus production amount and potential revenues) }\end{array}$ \\
\cline { 2 - 2 } $\begin{array}{l}\text { Scenario specific } \\
\text { modeling } \\
\text { requirements from } \\
\text { BtB case studies } \\
\text { the final product (affecting waste valorization) }\end{array}$ & Chemicals and energy use in bio-refineries (increasing environmental impacts) \\
\hline & Renewable energy sources use for energy generation (reducing environmental impacts) \\
\hline $\begin{array}{l}\text { Allocation rules in agricultural waste valorization: process infrastructures are out of the } \\
\text { scope }\end{array}$ & $\begin{array}{l}\text { Carbon imbalances are cut-off; direct pollutant emissions from UoL are avoided, but } \\
\text { generate impacts on the soil organic matter and nutrients cycling }\end{array}$ \\
\hline Combustion ashes are either waste or product, depending on their market value \\
\hline
\end{tabular}




High environmental impacts due to: cultivation-related land use, fertilizers production (fuel
consumption) and application (emissions), biomass drying and wood pelleting (fuel
consumption), pellets combustion (emissions)
Most impacted categories: human toxicity, freshwater eutrophication and natural land
transformation

The benefit of social-ecological systems (SES), such as agro-ecosystems, from such a modeling and management perspective is immediate, but the urban and industrial ecosystems can also take advantage from their representation as interacting cultural-biological entities (e.g. assemblages of species), even if they are dominated by a cultural (social-economic) determinism.

One of the challenges of the modeling efforts will be to integrate both abstract modeling requirements and concrete findings (case study-based), as resulted from the literature review. In the chosen context, the model's testing ground will be the biomass-to-biofuels processes, with the aim to identify, by a consequential approach, the mutual effects of the "resources" and "users" systems in the considered SES, and the applicable sustainability management constraints. Biodiversity and social-economic diversity (e.g. professional) will be represented as impact categories, which enables the operationalization of the resilience concept within the LCA framework, but the impacts breakdown by existing mid- and end-point categories will also be possible via the class-based representation of the processes assigned to the interacting species of the system. LCA flows inventory will integrate both transactions and relations (in particular immaterial cultural causalities), which can enhance the system boundaries and allocation rules definitions.

\section{CONCLUSIONS}

The current study analysis of the solutions' state of the art and of the gaps in the LCSA and LCA methodologies led to extracting and synthesizing some of the most promising models and of multi-domain, multi-criteria analytical concepts and methods applicable for a consistent evaluation of the systems' sustainability, with reference to the bioenergy context. Based on the state of the art solutions and identified gaps, considered as requirements, a "total ecosystem model" (TEM) will be fully specified in a future work, including a conceptual model for an extended ecosystem and two scorecards containing metrics for sustainability and fitness and measuring the ecosystem's performance in terms of "naturalness". The model will be enhanced with the requirements synthesized by the current literature review.

The expected results will account for the co-evolution feasibility between biological and competing cultural species, in various SESs, under sustainability constraints. As an example, in a BtB system, during the lignocellulosic biomass waste extraction from nature, in the biofuel value chain, detritivores and decomposers (involved in organic matter and minerals recycling) in soil ecosystems or trees and associated species in forest ecosystems interact with professional species like harvesters, transporters, collectors, chemists, fuel or energy distributors, ending with the "omnivorous" final consumers (key for the social reciprocation and markets stability). In a constructivist approach, certain processes will need to be adjusted, segregated within, added to or eliminated from the SES for improving its aggregated sustainability (e.g. its autonomy, via circularity enhancement, or viability and persistence, via resilience enhancement). The system evolution towards sustainable states can be realized by adaptation (e.g. applying preservation techniques, market restructuring) or by speciation (e.g. creating ecosystem conservation-related professions or introducing cleaner innovative technologies). Most of the processes mutations will involve selection on their carrying species (determining associated trophic and non-trophic interactions), but SESs management should avoid operating on the natural ecosystems' species, by massively adapting the anthropic cultural species to the natural processes.

Such a model of an extended ecosystem will reveal an incompatibility of the monetary valuation (used by the conventional cost-benefit analysis) with the sustainability definitions and its evaluation objectives. Simultaneously, the model will seek for optimization currencies reflecting intrinsic natural and cultural value creation, destruction and distribution along the matter-energy-information flux throughout the SES's network (associated to the focal product and productive processes), from a causal perspective. Therefore, an integration of the consequential LCA models with the orientors (goal functions, even based on qualitative metrics) resulting from an extended ecosystem-based sustainability model will bring homogeneity, objectivity and causal "defragmentation" to the networks under the LCSA scrutiny. A first step towards the integration is to create the abstract classes and metadata into the extended ecosystem model, able to capture the requirements resulting from the state-of-the-art LCA models scanning and to specify the adapted analytical methods and communication interfaces with the constructs used by the existing sustainability management landscape.

Author Contributions: E.K. Conceived, designed and wrote the paper; L.S. Collected the literature data; M-A.H. Contributed to the analytical framework; C.R. Supervised the work; D.E.D. Reviewed and validated the paper. 
Funding Source: This research was funded by the (1) Romanian Ministry of Research and Innovation, CCCDIUEFISCDI, PN-III-P1-1.2-PCCDI-2017 0251/4PCCDI/2018, and (2) PROINSTITUTIO project, 19PFE/2018.

\section{Acknowledgments}

This work was supported by: (1) a grant of the Romanian Ministry of Research and Innovation, CCCDI-UEFISCDI [project number PN-III-P1-1.2-PCCDI-2017 0251/4PCCDI/2018], (2) PROINSTITUTIO project [contract no. 19PFE/2018].

\section{Conflicts of Interest}

The authors declare that they do not have any conflict of interest.

\section{REFERENCES}

1. Bastianoni S (2002). Use of thermodynamic orientors to assess the efficiency of ecosystems: A case study in the Lagoon of Venice. The Scientific World Journal, 2: 255-260.

2. Benetto E, Jury C, Kneip G, Vazquez-Rowe I, Huck V, Minette F (2015). Life cycle assessment of heat production from grape marc pellets. Journal of Cleaner Production, 87: 149-158.

3. Brown M.T., Ulgiati S (1999), Emergy evaluation of the biosphere and natural capital. Ambio, 28(6): 486-493.

4. Ekener E, Hansson J, Larsson A, Peck P (2018). Developing life cycle sustainability assessment methodology by applying values-based sustainability weighting - Tested on biomass based and fossil transportation fuels. Journal of Cleaner Production, 181: 337-351.

5. Fantozzi F, Buratti C (2010). Life cycle assessment of biomass chains: Wood pellet from short rotation coppice using data measured on a real plant. Biomass and Bioenergy, 34(12): 1796-1804.

6. Gullon P, Gullon B, Davila I, Labidi J, Gonzalez-Garcia S (2018). Comparative environmental Life Cycle Assessment of integral revalorization of vine shoots from a biorefinery perspective. Science of the Total Environment, 624: 225-240.

7. Gulotta TM., Guarino F, Mistretta M, Cellura M, Lorenzini G (2018). Introducing exergy analysis in life cycle assessment: A case study. Mathematical Modelling of Engineering Problems, 5(3): 139-145.

8. ISO 14040 (2006). Environmental management-Life cycle assessment-Principles and framework.

9. Kylili A, Christoforou E, Fokaides PA (2016). Environmental evaluation of biomass pelleting using life cycle assessment. Biomass and Bioenergy, 84: 107-117.

10. Lifset R, Graedel TE (2015). Industrial Ecology. International Encyclopedia of the Social \& Behavioral Sciences, 2: 843-853.

11. Marvuglia A, Benetto E, Rios GP, Rugani B (2013). SCALE: Software for CALculating Emergy based on life cycle inventories, Ecological Modelling, 248(1): 80-91.

12. McManus MC, Taylor CM (2015). The changing nature of life cycle assessment. Biomass Bioenergy, 82: 13-26.

13. Millward-Hopkins J, Busch J, Purnell P, Zwirner O (2018). Fully integrated modelling for sustainability assessment of resource recovery from waste. Science in the Total Environment, 612: 613-624.

14. Roos A, Ahlgren S (2018). Consequential life cycle assessment of bioenergy systems - A literature review. Journal of Cleaner Production, 189: 358-373.

15. Schiller F, Alexandra SP, Basson L (2014). Analyzing networks in industrial ecology - A review of social-material network analyses. Journal of Cleaner Production, 76: 1-11.

16. Zvolinschi A, Kjelstrup S, Bolland O, van der Kooi HJ (2007). Exergy sustainability indicators as a tool in industrial ecology. Journal of Industrial Ecology, 85-98. 